\title{
Asymptotic normality of the likelihood moment estimators for a stationary linear process with heavy-tailed innovations
}

\author{
Lukas Martig · Jürg Hüsler
}

Received: 25 Mai 2016 / Accepted: date

\begin{abstract}
A variety of estimators for the parameters of the Generalized Pareto distribution, the approximating distribution for excesses over a high threshold, have been proposed, always assuming the underlying data to be independent. We recently proved in 9] that the likelihood moment estimators are consistent estimators for the parameters of the Generalized Pareto distribution for the case where the underlying data arises from a (stationary) linear process with heavy-tailed innovations. In this paper we derive the bivariate asymptotic normality under some additional assumptions and give an explicit example on how to check these conditions by using asymptotic expansions.
\end{abstract}

Keywords Generalized Pareto distribution · Linear processes · Heavy-tailed data $\cdot$ Likelihood moment estimators · Asymptotic Normality

Mathematics Subject Classification (2010) 60G50 - 60G70 - 62G20 • $62 \mathrm{G} 32$

1 Fitting a Generalized Pareto distribution for a linear process with regularly varying tails

We consider a (strictly) stationary linear process

$$
X_{n}=\sum_{j=0}^{\infty} c_{j} Z_{n-j}
$$

L. Martig

University of Bern, Inst. math. Statistics

Sidlerstrasse 5, 3012 Bern, Switzerland

Tel.: +41-31-6318811

Fax: +41-31-6313870

E-mail: lukasmartig@gmail.com

J. Hüsler

Inst. math. Statistics

Sidlerstrasse 5, 3012 Bern, Switzerland 
whose iid innovations $Z_{n}$ have a marginal distribution function $G_{Z}$ with regularly varying (i.e heavy) tails of index $-1 / \gamma$, for $\gamma>0$, i.e.

$$
1-G_{Z}(z) \sim \pi_{1} z^{-1 / \gamma} L(z) \text { and } G_{Z}(-z) \sim \pi_{2} z^{-1 / \gamma} L(z) \quad \text { as } z \rightarrow \infty,
$$

for $\pi_{1}, \pi_{2} \geq 0, \pi_{1}+\pi_{2}=1$ with a slowly varying function $L(z)$, where $\left\{c_{j}\right\}_{j \geq 0} \in \mathbb{R}^{\infty}$. Clearly, A.2 implies $1-G_{|Z|}(z) \sim z^{-1 / \gamma} L(z)$, where $G_{|Z|}$ is the (marginal) distribution function of $\left|Z_{n}\right|$.

Furthermore, for $\left\{c_{j}\right\}_{j \geq 0}$ being the sequence of coefficients in (A.1), let's assume that there is at least one $c_{j} \neq 0$ and there exist $A>0$ and $u>1$ such that

$$
\left|c_{j}\right|<A u^{-j}, \quad j \in \mathbb{N}_{0} .
$$

Here, it is worth to mention that every causal $\operatorname{ARMA}(p, q)$ process of the form

$$
X_{n}-\phi_{1} X_{n-1}-\ldots-\phi_{p} X_{n-p}=Z_{n}+\theta_{1} Z_{n-1}+\ldots+\theta_{q} Z_{n-q},
$$

$\phi_{1}, \ldots, \phi_{p} ; \theta_{1}, \ldots, \theta_{q} \in \mathbb{R}$, has a representation as in (A.1) such that (A.3) automatically holds (2], p. 85). From now on, A.1 - A.3 will be considered as Assumptions 1-3.

If we denote the marginal distribution function of $\left|X_{n}\right|$ by $\mathrm{F}_{|X|}$, then, with the help of Lemma 5.2 of [3], we directly conclude that the Assumptions 1-3 imply

$$
\lim _{t \rightarrow \infty} \frac{1-\mathrm{F}_{|X|}(t)}{1-\mathrm{G}_{|Z|}(t)}=\sum_{k=0}^{\infty}\left|c_{k}\right|^{1 / \gamma}:=\|c\| .
$$

Under mild restrictions on the coefficients $c_{j}$ the tail behavior of $\mathrm{F}_{|X|}$ thus coincides with that of $\mathrm{G}_{|Z|}$ up to the constant $\|c\|$ and consequently, the marginal distribution of the time series $\left|X_{n}\right|$ has also a regularly varying tail of index $-1 / \gamma$. According to [11], Theorem 7, it then follows that there exists a positive function $\sigma^{*}(t)$ such that for $\mathrm{F}_{|X|, t}(x):=\mathrm{P}\left(\left|X_{n}\right|-t \leq x|| X_{n} \mid>t\right)$ :

$$
\lim _{t \rightarrow \infty} \sup _{x>0}\left|\mathrm{~F}_{|X|, t}(x)-\left[1-\left(1+\frac{\gamma}{\sigma^{*}(t)} x\right)^{-1 / \gamma}\right]\right|=0 .
$$

The function $\mathrm{H}_{\gamma, \sigma^{*}}(x)=1-\left(1+\gamma x / \sigma^{*}(t)\right)^{-1 / \gamma}$ is known as the Generalized Pareto distribution (GPD) with scale function $\sigma^{*}(t)$. (In fact, if $\gamma>0$, the GPD simply equals to the Pareto distribution.) Relation (1.2) thus tells us, that the random variable $\left|X_{n}\right|-t|| X_{n} \mid>t$, the excess above a high threshold $t$, has approximately a Generalized Pareto distribution. In practice, $t$ is replaced by the $(k+1)$ th largest observation $|X|_{n, n-k}$ for a sufficiently large $k<<n$, i.e. $k=k(n)=o(n)$. Consequently, the target will be to estimate $\gamma$ and $\sigma^{*}(t)$ using the $k$ excess-values $|X|_{n, n}-|X|_{n, n-k}, \ldots,|X|_{n, n-k+1}-|X|_{n, n-k}$.

A variety of estimators have been proposed for the two parameters $\gamma$ and $\sigma^{*}=\sigma^{*}(t)$ such as the maximum likelihood estimators ([16]), the moment and 
probability weighted moment estimators ([5]), the likelihood moment estimators ([17]) or the goodness-of-fit estimators ([7]), to mention only a few. A nice résumé is also available in [4].

We showed in [9] that the likelihood moment estimators $\left(\hat{\gamma}_{L M E}, \hat{\sigma}_{L M E}\right)^{T}$, the solutions of the following system of equations for $\gamma$ and $\sigma^{*}$ :

$$
\begin{aligned}
& \frac{1}{k} \sum_{i=0}^{k-1} \log \left(1+\frac{\gamma}{\sigma^{*}}\left(|X|_{n, n-i}-|X|_{n, n-k}\right)\right)=\gamma \\
& \frac{1}{k} \sum_{i=0}^{k-1}\left(1+\frac{\gamma}{\sigma^{*}}\left(|X|_{n, n-i}-|X|_{n, n-k}\right)\right)^{r / \gamma}=(1-r)^{-1},
\end{aligned}
$$

are consistent estimators for the parameters of the Generalized Pareto distribution if A.1 -A.3 hold and $r<0$. In this paper, we prove the asymptotic bivariate normality of $\left(\hat{\gamma}_{L M E}, \hat{\sigma}_{L M E}\right)^{T}$ under some additional assumptions, see Theorem 7 and Corollary 8 in Section 2,

$$
\sqrt{k}\left(\begin{array}{c}
\hat{\gamma}_{L M E}-\gamma \\
\frac{\hat{\sigma}_{L M E}}{\sigma(n / k)}-1
\end{array}\right) \stackrel{D}{\rightarrow} N\left(\mathbf{0}, L \Sigma L^{T}\right) .
$$

\section{Asymptotic normality of $\left(\hat{\gamma}_{L M E}, \hat{\sigma}_{L M E}\right)^{T}$}

In this section we prove asymptotic bivariate normality of $\left(\hat{\gamma}_{L M E}, \hat{\sigma}_{L M E}\right)^{T}$. Thereby, we will use and also extend some of the results about tail array sums of 14 and [15. For the calculation of the covariance matrix, the methods of 13. are applied.

For our result we need further assumptions. Throughout, the sequence $k=$ $k(n), k=o(n)$ refers to the $(k+1)$ th largest observation $|X|_{n, n-k}$ and for simplicity, we will assume that there exist no ties and that $|X|_{n, n-k}$ is uniquely defined (notice that - under some weak additional conditions - all results surely continue to hold in the presence of ties, see Remark 4.4 in [14). Also, we assume $r$ in (1.4) to be negative and finally, for $b_{|X|}(t):=\left(1 /\left(1-\mathrm{F}_{|X|}\right)\right)^{\leftarrow}(t):=\inf \{y:$ $\left.1 /\left(1-\mathrm{F}_{|X|}(y)\right) \geq t\right\}$ being the $1-1 / t$-quantile of $\mathrm{F}_{|X|}$, let's define

$$
\sigma(t):=\sigma^{*}\left(b_{|X|}(t)\right)
$$

so that for any $x>0$ as $t \rightarrow \infty$ (see 9 ):

$$
\frac{b_{|X|}(t x)-b_{|X|}(t)}{\sigma(t)} \rightarrow \frac{x^{\gamma}-1}{\gamma} .
$$


Definition 1 Let $\mathfrak{B}_{i, j}$ be the $\sigma$-field $\sigma\left(X_{h}\right)_{i \leq h \leq j}$ generated by a sequence $X_{i}, X_{i+1}, \ldots, X_{j}$. Then for fixed $n, l \in \mathbb{N} \backslash\{0\}$ with $l<n$ we define

$\alpha_{n, l}:=\sup \left(|\mathrm{P}(A \cap B)-\mathrm{P}(A) \mathrm{P}(B)|: A \in \mathfrak{B}_{1, h}, B \in \mathfrak{B}_{h+l, n}, 1 \leq h \leq n-l\right)$,

and we say that $\left\{X_{j}\right\}$ is $\left(\alpha_{n, l_{n}}, l_{n}\right)$-strongly mixing if $\alpha_{n, l_{n}} \rightarrow 0$ for some $l_{n} \rightarrow \infty$ with $l_{n}=o(n)$.

Definition 2 Let $b_{|X|}(t)$ and $\sigma(t)$ be defined as in (2.1), then we say $b_{|X|}$ is second-order extended regularly varying, $2 \operatorname{ERV}(\rho, A)$ for short, if for $x>0$, $\rho \leq 0, \gamma \neq-\rho$, there exists a positive (or negative) function $A(t)$ of constant sign near infinity, $A(t) \rightarrow 0$ as $t \rightarrow \infty$, such that

$$
\lim _{t \rightarrow \infty} \frac{\frac{b_{|X|}(t x)-b_{|X|}(t)}{\sigma(t)}-\frac{x^{\gamma}-1}{\gamma}}{A(t)}=\frac{1}{\rho}\left(\frac{x^{\gamma+\rho}-1}{\gamma+\rho}-\frac{x^{\gamma}-1}{\gamma}\right)
$$

and

$$
\lim _{n, k, n / k \rightarrow \infty} \sqrt{k} A(n / k)=0 .
$$

Definition $31-\mathrm{F}_{|X|}$ is said to be second-order regularly varying, $2 \mathrm{RV}\left(\rho^{\prime}, A^{*}\right)$ for short, if for $x>0, \rho^{\prime} \leq 0, \gamma \neq-\rho^{\prime}$, there exists a positive (or negative) function $A^{*}(t)$ of constant sign near infinity, $A^{*}(t) \rightarrow 0$ as $t \rightarrow \infty$, such that

$$
\lim _{t \rightarrow \infty} \frac{\frac{1-\mathrm{F}_{|X|}(t x)}{1-\mathrm{F}_{|X|}(t)}-x^{-1 / \gamma}}{A^{*}(t)}=x^{-1 / \gamma} \frac{x^{\rho^{\prime}}-1}{\rho^{\prime}}
$$

and

$$
\lim _{n, k, n / k \rightarrow \infty} \sqrt{k} A^{*}\left(b_{|X|}(n / k)\right) \rightarrow 0 .
$$

The following assumptions will be of prime importance throughout this section:

Let $\left\{c_{j}\right\}_{j \geq 0}$ be the sequence of coefficients in (A.1). Then we assume that

$$
\sum_{j=1}^{\infty} \sum_{i=0}^{\infty}\left(\left|c_{i}\right| \wedge\left|c_{i+j}\right|\right)^{1 / \gamma} \log \left(\frac{\left|c_{i}\right| \vee\left|c_{i+j}\right|}{\left|c_{i}\right| \wedge\left|c_{i+j}\right|}\right) \mathbf{1}\left\{c_{i} c_{i+j} \neq 0\right\}<\infty
$$

The sequence of thresholds $u_{n}$ is chosen such that $n\left(1-\mathrm{F}_{|X|}\left(\exp \left(u_{n}\right)\right)\right)=k+1$.

$\left\{X_{j}\right\}$ is $\left(\alpha_{n, l_{n}}, l_{n}\right)$-strongly mixing.

A sequence of integers $r_{n}$ with $l_{n}<r_{n} \leq n$ is chosen such that $k r_{n}=o(n)$ and for $m_{n}:=\left[n / r_{n}\right]$ we have $m_{n}\left(\alpha_{n, l_{n}}+l_{n} / n\right) \rightarrow 0$ as $n \rightarrow \infty$. 
$\mathrm{F}_{|X|}$ has a derivative $\mathrm{F}_{|X|}^{\prime}$ in $\left(t_{0}, \infty\right)$ for some $t_{0}>0$ and we assume that

$$
\lim _{t \rightarrow \infty} \frac{t \mathrm{~F}_{|X|}^{\prime}(t)}{1-\mathrm{F}_{|X|}(t)}=\frac{1}{\gamma}
$$

$b_{|X|} \in 2 \operatorname{ERV}(\rho, A)$ and $1-\mathrm{F}_{|X|} \in \operatorname{RV}\left(\rho^{\prime}, A^{*}\right)$.

The properties (A.4)-(A.9) will be called Assumptions 4-9. Notice that the sequence $r_{n}$ in (A.7) always exists (e.g. $\left.r_{n}=\left\lceil\max \left(n \alpha_{n, l_{n}}^{1 / 2}, n^{3 / 2} l_{n}^{-1 / 2}\right)\right\rceil\right)$. The sequence $m_{n}$ is called the standard sequence which decomposes $(0, n]$ into intervals $J_{i}:=\left((i-1) r_{n}, i r_{n}\right], 1 \leq i \leq m_{n}$ and a last interval $J_{m_{n}+1}:=$ $\left(m_{n} r_{n}, n\right]$ termed the standard partition. Finally, one can show that if $0<$ $-\rho<\gamma$, then $b_{|X|} \in 2 \operatorname{ERV}(\rho, A)$ implies $1-\mathrm{F}_{|X|} \in \operatorname{RV}\left(\rho^{\prime}, A^{*}\right)$ with $\rho^{\prime}=\rho / \gamma$ and $A^{*}(t)=A\left(1 /\left(1-\mathrm{F}_{|X|}(t)\right)\right) /\left(\gamma^{2}\left(1+\rho^{\prime}\right)\right)$. In case $0<\gamma<-\rho$ the additional assumption $\lim _{t \rightarrow \infty} \gamma b_{|X|}(t)-\sigma(t)=0$ is needed ([10], Theorem 1, and [4], Remark 2.3.10).

Now, since $1-\mathrm{F}_{|X|}$ is regularly varying, it directly follows by (A.5) that for any $x>0$ :

$$
\frac{n}{k}\left(1-\mathrm{F}_{|X|}\left(x \exp \left(u_{n}\right)\right)\right) \sim \frac{1-\mathrm{F}_{|X|}\left(x \exp \left(u_{n}\right)\right)}{1-\mathrm{F}_{|X|}\left(\exp \left(u_{n}\right)\right)} \sim x^{-1 / \gamma}
$$

as $n, k, n / k \rightarrow \infty$, which can be extended by an application of Lemma 5.1 and Lemma 5.2 of [3] to

$$
\begin{aligned}
\frac{n}{k} \mathrm{P} & \left(\left|X_{1}\right|>x \exp \left(u_{n}\right),\left|X_{j+1}\right|>y \exp \left(u_{n}\right)\right) \\
& \sim \frac{1}{\|c\|} \sum_{k=0}^{\infty}\left(\left|c_{k}\right|^{1 / \gamma} x^{-1 / \gamma} \wedge\left|c_{j+k}\right|^{1 / \gamma} y^{-1 / \gamma}\right)
\end{aligned}
$$

locally uniformly in $x, y \in(0, \infty)$, where any $j \in \mathbb{N}_{0}$.

Next consider the two functions

$$
\phi_{1}(x):=x \mathbf{1}\{x>0\} \text { and } \phi_{2}(x):=(1-\exp (r x / \gamma)) \mathbf{1}\{x>0\},
$$

where $r<0$. As we will see later on, there exists a clear connection between the tail array sums involved in the likelihood moment estimation (i.e. (1.3) and (1.4) $)$ and the ones implied by the functions $\phi_{1}$ and $\phi_{2}$ when considering the transformed sequence $\left\{\log \left(\left|X_{j}\right|\right)\right\}$. Furthermore, the properties in (2.2) and (2.3) are such that they allow us to use and slightly modify the results of Resnick \& Stâricâ in [13]. After some repetition, it is evident that their convergences stated in Lemma 2.1 and Remark 2.1 as well as their methods applied in the respective proofs continue to hold with $b_{|X|}(n / k)$ replaced by our $\exp \left(u_{n}\right)$ and this allows us to calculate the means, the variances and the covariances of tail array block-sums based on $\phi_{i}\left(\log \left(\left|X_{j}\right| / \exp \left(u_{n}\right)\right)\right)=\phi_{i}\left(\log \left(\left|X_{j}\right|\right)-u_{n}\right), i=$ 1,2 . 
Throughout, we will use the following notations for $Y_{j}:=\log \left(\left|X_{j}\right| / \exp \left(u_{n}\right)\right)$, $i=1,2$ and $r<0$ :

$$
\begin{aligned}
\beta_{1}^{\prime} & :=\frac{\gamma}{(\gamma+1)}, \quad \beta_{2}^{\prime}:=-\frac{r}{(1-r+\gamma)} \text { and } \tilde{\phi}_{i}(x):=\phi_{i}(x)-\beta_{i}^{\prime} \mathbf{1}\{x>0\} \\
\left(\tau_{n}^{(i)}\right)^{2} & :=\frac{n}{r_{n}} \operatorname{Var}\left(\sum_{j=1}^{r_{n}} \phi_{i}\left(Y_{j}\right)\right), \quad\left(\tau_{n}^{(I)}\right)^{2}:=\frac{n}{r_{n}} \operatorname{Var}\left(\sum_{j=1}^{r_{n}} \mathbf{1}\left\{Y_{j}>0\right\}\right), \\
\tau_{n}^{(1,2)} & :=\frac{n}{r_{n}} \operatorname{Cov}\left(\sum_{j=1}^{r_{n}} \phi_{1}\left(Y_{j}\right), \sum_{j=1}^{r_{n}} \phi_{2}\left(Y_{j}\right)\right) \\
\tau_{n}^{(i, I)} & :=\frac{n}{r_{n}} \operatorname{Cov}\left(\sum_{j=1}^{r_{n}} \phi_{i}\left(Y_{j}\right), \sum_{j=1}^{r_{n}} \mathbf{1}\left\{Y_{j}>0\right\}\right), \\
\left(\tilde{\tau}_{n}^{(i)}\right)^{2} & :=\frac{n}{r_{n}} \operatorname{Var}\left(\sum_{j=1}^{r_{n}} \tilde{\phi}_{i}\left(Y_{j}\right)\right), \quad \tilde{\tau}_{n}^{(1,2)}:=\frac{n}{r_{n}} \operatorname{Cov}\left(\sum_{j=1}^{r_{n}} \tilde{\phi}_{1}\left(Y_{j}\right), \sum_{j=1}^{r_{n}} \tilde{\phi}_{2}\left(Y_{j}\right)\right) .
\end{aligned}
$$

Finally, for $\left\{c_{j}\right\}_{j \geq 0}$ being again the sequence of coefficients in (A.1), we define

$$
\begin{aligned}
\varphi_{1} & :=\frac{1}{\| c||} \sum_{j=1}^{\infty} \sum_{k=0}^{\infty}\left(\left|c_{k}\right| \wedge\left|c_{j+k}\right|\right)^{1 / \gamma}, \\
\varphi_{2} & :=\frac{1}{\| c||} \sum_{j=1}^{\infty} \sum_{k=0}^{\infty} \frac{\left(\left|c_{k}\right| \vee\left|c_{j+k}\right|\right)^{r / \gamma}}{\left(\left|c_{k}\right| \wedge\left|c_{j+k}\right|\right)^{(r-1) / \gamma}} \mathbf{1}\left\{c_{k}, c_{j+k} \neq 0\right\}, \\
\varphi_{3} & :=\frac{1}{\|c\|} \sum_{j=1}^{\infty} \sum_{k=0}^{\infty}\left(\left|c_{k}\right| \wedge\left|c_{j+k}\right|\right)^{1 / \gamma} \log \left(\frac{\left|c_{k}\right| \vee\left|c_{j+k}\right|}{\left|c_{k}\right| \wedge\left|c_{j+k}\right|}\right) \mathbf{1}\left\{c_{k}, c_{j+k} \neq 0\right\} .
\end{aligned}
$$

Result 1 Let the Assumptions 1-5 hold. Then for a sequence of integers $r_{n} \rightarrow$ $\infty$ satisfying $k r_{n}=o(n)$ we have as $n, k, n / k \rightarrow \infty$ :

$$
\begin{aligned}
& \frac{\left(\tau_{n}^{(1)}\right)^{2}}{k} \rightarrow 2 \gamma\left(\gamma+2 \gamma \varphi_{1}+\varphi_{3}\right), \\
& \frac{\left(\tau_{n}^{(2)}\right)^{2}}{k} \rightarrow \frac{-2 r\left(-r+(1-2 r) \varphi_{1}-\varphi_{2}\right)}{(1-r)(1-2 r)}, \\
& \frac{\left(\tau_{n}^{(I)}\right)^{2}}{k} \rightarrow 1+2 \varphi_{1},
\end{aligned}
$$




$$
\begin{aligned}
& \frac{\tau_{n}^{(1,2)}}{k} \rightarrow \frac{\left(-\gamma r(2-r)+\left(2 r^{2}-4 r+1\right) \gamma \varphi_{1}-\gamma \varphi_{2}-r(1-r) \varphi_{3}\right)}{(1-r)^{2}} \\
& \frac{\tau_{n}^{(1, I)}}{k} \rightarrow \gamma+2 \gamma \varphi_{1}+\varphi_{3} \\
& \frac{\tau_{n}^{(2, I)}}{k} \rightarrow \frac{\left(-r+(1-2 r) \varphi_{1}-\varphi_{2}\right)}{(1-r)}, \\
& \frac{\left(\tilde{\tau}_{n}^{(1)}\right)^{2}}{k} \rightarrow \frac{\left(1+2 \varphi_{1}\right) \gamma^{2}\left(2 \gamma^{2}+2 \gamma+1\right)+2 \gamma^{2}(\gamma+1) \varphi_{3}}{(\gamma+1)^{2}}:=\kappa_{1}(\gamma, r), \\
& \frac{\left(\tilde{\tau}_{n}^{(2)}\right)^{2}}{k} \rightarrow \frac{-2 \gamma r(\gamma+1)\left(-r+\varphi_{1}(1-2 r)-\varphi_{2}\right)+r^{2}(1-r)\left(1+2 \varphi_{2}\right)}{(1-r)(1-2 r)(1-r+\gamma)^{2}}:=\kappa_{2}(\gamma, r), \\
& \frac{\tilde{\tau}_{n}^{(1,2)}}{k} \rightarrow-\frac{\gamma r\left((2-r)\left(\gamma^{2}+\gamma+1\right)-1\right)}{(1-r)^{2}(1+\gamma)(1-r+\gamma)} \\
& -\frac{\gamma\left(2 r(2-r)\left(\gamma^{2}+\gamma+1\right)-\left(\gamma^{2}+\gamma+3 r-r^{2}\right)\right)}{(1-r)^{2}(1+\gamma)(1-r+\gamma)} \cdot \varphi_{1} \\
& -\frac{\gamma(\gamma+r)}{(1-r)^{2}(1+\gamma)} \cdot \varphi_{2}-\frac{\gamma r}{(1-r)(1-r+\gamma)} \cdot \varphi_{3}:=\kappa_{3}(\gamma, r) . \quad(2.14) \text { ?2.2.9.6? }
\end{aligned}
$$

Proof By virtue of (2.2) and (2.3), the convergences (2.6)-(2.14) directly follow after a small repetition of the proofs of Lemma 2.1 and Remark 2.1 in [13. with $b_{|X|}(n / k)$ replaced by our $\exp \left(u_{n}\right)$.

The next result shows the asymptotic behavior of $S_{n}^{(i)}(x):=\left(\sum_{j=1}^{n} \mathbf{1}\left\{\log \left(\left|X_{j}\right|\right)>x\right\}-n\left(1-\mathrm{F}_{|X|}(\exp (x))\right)\right) / \tilde{\tau}_{n}^{(i)}, i=1,2$.

Lemma 2 Suppose the Assumptions 1-8 hold. Additionally, let

$$
\frac{r_{n}}{\sqrt{k}} \rightarrow 0
$$

as $n, k, n / k \rightarrow \infty$. Then for $i=1,2$ :

$$
\begin{aligned}
& S_{n}^{(i)}\left(u_{n}\right) \stackrel{D}{\rightarrow} N\left(0,\left(1+2 \varphi_{1}\right) / \kappa_{i}(\gamma, r)\right), \\
& S_{n}^{(i)}\left(\log \left(|X|_{n, n-k}\right)\right)-S_{n}^{(i)}\left(u_{n}\right) \stackrel{P}{\rightarrow} 0, \\
& k\left(\log \left(|X|_{n, n-k}\right)-u_{n}\right) / \tilde{\tau}_{n}^{(i)}-\gamma S_{n}^{(i)}\left(u_{n}\right) \stackrel{P}{\rightarrow} 0 .
\end{aligned}
$$

Proof Let $i=1$ and recall $Y_{j}=\log \left(\left|X_{j}\right| / \exp \left(u_{n}\right)\right)$. By definition

$$
S_{n}^{(1)}\left(u_{n}\right)=\frac{\sum_{j=1}^{n}\left[\mathbf{1}\left\{Y_{j}>0\right\}-\mathrm{E}\left(\mathbf{1}\left\{Y_{1}>0\right\}\right)\right]}{\tau_{n}^{(I)}} \cdot \frac{\tau_{n}^{(I)}}{\tilde{\tau}_{n}^{(1)}}:=\mathrm{I}_{1} \cdot \mathrm{I}_{2} .
$$


Using all our assumptions, it is straightforward that all conditions of Theorem 6.2 of [15] hold for the transformed sequence $\left\{\log \left(\left|X_{j}\right|\right)\right\}$ and tail array function $\phi(x)=\mathbf{1}\{x>0\}$ and this simply means that $\mathrm{I}_{1} \stackrel{D}{\rightarrow} N(0,1)$ as $n, k, n / k \rightarrow \infty$. With the help of Result 1 it is then easy to see that $\mathrm{I}_{2} \rightarrow \sqrt{\left(1+2 \varphi_{1}\right) / \kappa_{1}(\gamma, r)}$ as $n, k, n / k \rightarrow \infty$. This shows (2.16) for $i=1$.

To show (2.17) and (2.18), it suffices to check whether all conditions of Lemma 4.2 of 14 are again satisfied for $\left\{\log \left(\left|X_{j}\right|\right)\right\}$ and the task then reduces to prove that for any non-random sequence $z_{n}$ with $k\left(z_{n}-u_{n}\right) / \tilde{\tau}_{n}^{(1)}$ bounded we have $S_{n}^{(1)}\left(z_{n}\right)-S_{n}^{(1)}\left(u_{n}\right) \stackrel{P}{\rightarrow} 0$ as $n, k, n / k \rightarrow \infty$. By the very same paper (pp. 21$23)$, this condition is satisfied if for $v_{n}:=\exp \left(z_{n}\right), I_{n}:=\left[u_{n}, \log \left(v_{n}\right)\right)$ in case $\log \left(v_{n}\right)>u_{n}$ and $I_{n}:=\left[\log \left(v_{n}\right), u_{n}\right)$ in case $\log \left(v_{n}\right)<u_{n}$ :

$$
\frac{n}{r_{n}\left(\tilde{\tau}_{n}^{(1)}\right)^{2}} \operatorname{Var}\left(\sum_{j=1}^{r_{n}} \mathbf{1}\left\{\log \left(\left|X_{j}\right|\right) \in I_{n}\right\}\right) \rightarrow 0
$$

whenever $v_{n} / \exp \left(u_{n}\right) \rightarrow 1$ as $n, k, n / k \rightarrow \infty$. But going along the steps of the proof of Lemma 2.1 and Remark 2.1 of [13] (or see the proof of Lemma 6 below) the convergence in (2.19) is obvious.

For the case $i=2$ analogous considerations show the statements.

The next result shows the limit behavior of the difference of our selected tail array sums when two different thresholds are chosen: the random value $\log \left(|X|_{n, n-k}\right)$ in the first case and $u_{n}$ in the second case.

Result 3 Let Assumptions 1-8 and (2.15) hold. Recall that $Y_{j}=\log \left(\left|X_{j}\right|\right)-u_{n}$ and define $Y_{j}^{\prime}:=\log \left(\left|X_{j}\right|\right)-\log \left(|X|_{n, n-k}\right)$. Then for $r<0$ :

$$
\begin{aligned}
& \frac{1}{\tilde{\tau}_{n}^{(1)}} \sum_{j=1}^{n}\left[\phi_{1}\left(Y_{j}^{\prime}\right)-\phi_{1}\left(Y_{j}\right)\right]+\gamma S_{n}^{(1)}\left(u_{n}\right) \stackrel{P}{\rightarrow} 0, \\
& \frac{1}{\tilde{\tau}_{n}^{(2)}} \sum_{j=1}^{n}\left[\phi_{2}\left(Y_{j}^{\prime}\right)-\phi_{2}\left(Y_{j}\right)\right]-\frac{r S_{n}^{(2)}\left(u_{n}\right)}{(1-r)} \stackrel{P}{\rightarrow} 0,
\end{aligned}
$$

as $n, k, n / k \rightarrow \infty$.

Proof The proof of (2.20) was originally given by [14, pp. 15-19. Thus we will lay our focus on (2.21), which can be proved in a very similar way. To begin, let's retain from the aforementioned paper that as $n, k, n / k \rightarrow \infty$ :

$$
R_{n}^{(2)}:=\frac{1}{\tilde{\tau}_{n}^{(2)}} \sum_{j=1}^{n}\left(\log \left(\left|X_{j}\right|\right)-u_{n}\right) \mathbf{1}\left\{\log \left(|X|_{n, n-k}\right) \geq \log \left(\left|X_{j}\right|\right)>u_{n}\right\} \stackrel{P}{\rightarrow} 0,
$$

and similarly,

$(2.22)$ ? reminder1?

$T_{n}^{(2)}:=\frac{1}{\tilde{\tau}_{n}^{(2)}} \sum_{j=1}^{n}\left(\log \left(\left|X_{j}\right|\right)-\log \left(|X|_{n, n-k}\right)\right) \mathbf{1}\left\{u_{n} \geq \log \left(\left|X_{j}\right|\right)>\log \left(|X|_{n, n-k}\right)\right\} \stackrel{P}{\rightarrow} 0$.

(2.23) ? $\underline{\text { reminder2 }}$ ? 
Also, using standard arguments (9], Lemma 1, and [12], pp. 80-85), one quickly shows that

$$
\frac{1}{k} \sum_{j=0}^{k-1}\left(\frac{|X|_{n, n-j}}{|X|_{n, n-k}}\right)^{r / \gamma} \stackrel{P}{\rightarrow} \frac{1}{(1-r)}
$$

$n, k, n / k \rightarrow \infty$.

Now, let's consider the set $\left\{|X|_{n, n-k}>\exp \left(u_{n}\right)\right\}$. Usually, this can be done by multiplying $\mathbf{1}\left\{|X|_{n, n-k}>\exp \left(u_{n}\right)\right\}$ throughout, but for simplicity, we will not denote it and just assume $|X|_{n, n-k}>\exp \left(u_{n}\right)$ in the computations below (cf. [14]). Then

$$
\begin{aligned}
& \frac{1}{\tilde{\tau}_{n}^{(2)}} \sum_{j=1}^{n}\left[\phi_{2}\left(Y_{j}^{\prime}\right)-\phi_{2}\left(Y_{j}\right)\right] \\
= & \frac{1}{\tilde{\tau}_{n}^{(2)}} \sum_{j=0}^{k-1}\left[\left(\frac{|X|_{n, n-j}}{\exp \left(u_{n}\right)}\right)^{r / \gamma}-\left(\frac{|X|_{n, n-j}}{|X|_{n, n-k}}\right)^{r / \gamma}\right] \\
& -\frac{1}{\tilde{\tau}_{n}^{(2)}} \sum_{j=1}^{n}\left(1-\left(\frac{\left|X_{j}\right|}{\exp \left(u_{n}\right)}\right)^{r / \gamma}\right) \mathbf{1}\left\{\left|X_{n, n-k}\right| \geq\left|X_{j}\right|>\exp \left(u_{n}\right)\right\}:=\mathrm{I}_{1}+\mathrm{I}_{2} .
\end{aligned}
$$

Next, since $|X|_{n, n-k} / \exp \left(u_{n}\right) \stackrel{P}{\rightarrow} 1$ as $n, k, n / k \rightarrow \infty$ by (2.18) and $z^{r / \gamma}-1 \sim$ $r \log (z) / \gamma$ as $z \rightarrow 1$, an application of the continuous mapping theorem yields

$$
\begin{aligned}
\mathrm{I}_{1} & =\frac{1}{\tilde{\tau}_{n}^{(2)}} \sum_{j=0}^{k-1}\left(\frac{|X|_{n, n-j}}{|X|_{n, n-k}}\right)^{r / \gamma}\left(\left(\frac{|X|_{n, n-k}}{\exp \left(u_{n}\right)}\right)^{r / \gamma}-1\right) \\
& =\frac{r}{\gamma} \frac{k}{\tilde{\tau}_{n}^{(2)}}\left(\log \left(|X|_{n, n-k}\right)-u_{n}\right)\left(1+o_{P}(1)\right) \frac{1}{k} \sum_{j=0}^{k-1}\left(\frac{|X|_{n, n-j}}{|X|_{n, n-k}}\right)^{r / \gamma} \\
& =\frac{r}{\gamma(1-r)} \frac{k}{\tilde{\tau}_{n}^{(2)}}\left(\log \left(|X|_{n, n-k}\right)-u_{n}\right)\left(1+o_{P}(1)\right)=\frac{r}{(1-r)} S_{n}^{(2)}\left(u_{n}\right)+o_{P}(1),
\end{aligned}
$$

where (2.16), 2.18) and (2.24) were used in the last line.

Finally, a simple application of the mean value theorem shows that $\left|\mathrm{I}_{2}\right| \leq$ $-r R_{n}^{(2)} / \gamma$, but this simply means $\mathrm{I}_{2} \stackrel{P}{\rightarrow} 0$ as $n, k, n / k \rightarrow \infty$ by (2.22).

The same result holds on the set $\left\{|X|_{n, n-k}<\exp \left(u_{n}\right)\right\}$ using similar arguments as above, (2.23) and by noticing that $\frac{1}{k} \sum_{j=1}^{n} \mathbf{1}\left\{\left|X_{j}\right|>\exp \left(u_{n}\right)\right\} \stackrel{P}{\rightarrow} 1$ as $n, k, n / k \rightarrow \infty$.

The next step will be to give the link between the tail array sums related to the likelihood moment estimators and our tail array sums selected at the very beginning of the section. The crucial point here is to make use of both the second-order and the second-order extended regular variation introduced 
in Assumption 9. With the help of Lemma B.3.16 of [4, we quickly conclude that if $b_{|X|} \in 2 \operatorname{ERV}(\rho, A)$, then

$$
\frac{\gamma b_{|X|}(n / k)}{\sigma(n / k)}=1+o(1 / \sqrt{k})
$$

as $n, k, n / k \rightarrow \infty$. Furthermore, going along the steps of [6], pp. 1552-1554, we also deduce that if $1-\mathrm{F}_{|X|} \in \mathrm{RV}\left(\rho^{\prime}, A^{*}\right)$, then $1-\mathrm{F}_{|X|}\left(b_{|X|}(n / k)\right)=k(1+$ $o(1 / \sqrt{k})) / n$. But since we chose $u_{n}$ such that $1-\mathrm{F}_{|X|}\left(\exp \left(u_{n}\right)\right)=(k+1) / n$, it follows that

$$
\frac{1-\mathrm{F}_{|X|}\left(\exp \left(u_{n}\right)\right)}{1-\mathrm{F}_{|X|}\left(b_{|X|}(n / k)\right)}=1+o(1 / \sqrt{k})
$$

and thus $A^{*}\left(b_{|X|}(n / k)\right) \sim A^{*}\left(\exp \left(u_{n}\right)\right)$ and finally

$$
\sqrt{k}\left(\frac{n}{k} \mathrm{E}\left(\phi_{i}\left(Y_{1}\right)\right)-\beta_{i}\right) \rightarrow 0,
$$

for $i=1,2, \beta_{1}:=\gamma$ and $\beta_{2}:=-r /(1-r)$. In conclusion, considering Assumption 8 ,

$$
\begin{aligned}
\frac{1-\mathrm{F}_{|X|}\left(\exp \left(u_{n}\right)\right)}{1-\mathrm{F}_{|X|}\left(b_{|X|}(n / k)\right)}-1 & \sim \log \left(\frac{1-\mathrm{F}_{|X|}\left(\exp \left(u_{n}\right)\right)}{1-\mathrm{F}_{|X|}\left(b_{|X|}(n / k)\right)}\right) \\
& =\log \left(1-\mathrm{F}_{|X|}\left(\exp \left(u_{n}\right)\right)\right)-\log \left(1-\mathrm{F}_{|X|}\left(b_{|X|}(n / k)\right)\right) \\
& =-\int_{1}^{\exp \left(u_{n}\right) / b_{|X|}(n / k)} \frac{b_{|X|}(n / k) s \mathrm{~F}_{|X|}^{\prime}\left(s b_{|X|}(n / k)\right)}{1-\mathrm{F}_{|X|}\left(s b_{|X|}(n / k)\right)} \frac{d s}{s} \\
& \sim-\frac{1}{\gamma} \int_{1}^{\exp \left(u_{n}\right) / b_{|X|}(n / k)} \frac{d s}{s}=-\frac{1}{\gamma} \log \left(\exp \left(u_{n}\right) / b_{|X|}(n / k)\right) \\
& \sim \frac{1}{\gamma}\left(\frac{b_{|X|}(n / k)}{\exp \left(u_{n}\right)}-1\right),
\end{aligned}
$$

which simply means $b_{|X|}(n / k) / \exp \left(u_{n}\right)=1+o(1 / \sqrt{k})$ by virtue of (2.26) and thus, using (2.25),

$$
\frac{\gamma \exp \left(u_{n}\right)}{\sigma(n / k)}=1+o(1 / \sqrt{k}),
$$

as $n, k, n / k \rightarrow \infty$.

Result 4 Let the Assumptions 1-9 and (2.15) hold. Recall that $Y_{j}^{\prime}=\log \left(\left|X_{j}\right|\right)-$ $\log \left(|X|_{n, n-k}\right)$ and define $Y_{j}^{\prime \prime}:=|X|_{n, n-j}-|X|_{n, n-k}$. Then:

$$
\begin{aligned}
& \frac{1}{\tilde{\tau}_{n}^{(1)}} \sum_{j=0}^{k-1}\left[\log \left(1+\frac{\gamma Y_{j}^{\prime \prime}}{\sigma(n / k)}\right)-\phi_{1}\left(Y_{j}^{\prime}\right)\right]-\frac{\gamma^{2} S_{n}^{(1)}\left(u_{n}\right)}{(\gamma+1)} \stackrel{P}{\rightarrow} 0, \\
& \frac{1}{\tilde{\tau}_{n}^{(2)}} \sum_{j=0}^{k-1}\left[\left(1+\frac{\gamma Y_{j}^{\prime \prime}}{\sigma(n / k)}\right)^{r / \gamma}-\left(1-\phi_{2}\left(Y_{j}^{\prime}\right)\right)\right]-\frac{\gamma r S_{n}^{(2)}\left(u_{n}\right)}{(1-r)(1-r+\gamma)} \stackrel{P}{\rightarrow} 0,
\end{aligned}
$$


as $n, k, n / k \rightarrow \infty$.

Proof With the help of 9], Lemma 1, and (2.25) it is easy to see that for any $j<k$ we always have

$$
\frac{|X|_{n, n-k}}{|X|_{n, n-j}}=O_{P}(1) \quad \text { and } \quad \frac{\gamma|X|_{n, n-k}}{\sigma(n / k)} \stackrel{P}{\rightarrow} 1
$$

as $n, k, n / k \rightarrow \infty$. To prove (2.29), notice that

$$
\begin{aligned}
& \frac{1}{\tilde{\tau}_{n}^{(1)}} \sum_{j=0}^{k-1}\left[\log \left(1+\frac{\gamma Y_{j}^{\prime \prime}}{\sigma(n / k)}\right)-\phi_{1}\left(Y_{j}^{\prime}\right)\right] \\
= & \frac{1}{\tilde{\tau}_{n}^{(1)}} \sum_{j=0}^{k-1} \log \left(\frac{|X|_{n, n-k}}{|X|_{n, n-j}}\left(1-\frac{\gamma|X|_{n, n-k}}{\sigma(n / k)}\right)+\frac{\gamma|X|_{n, n-k}}{\sigma(n / k)}\right):=\frac{1}{\tilde{\tau}_{n}^{(1)}} \sum_{j=0}^{k-1} \log \left(A_{j, k, n}\right) .
\end{aligned}
$$

Now, due to (2.31), $A_{j, k, n}=1+o_{P}(1)$ uniformly for $j<k$ as $n, k, n / k \rightarrow \infty$ and hence, using the fact that $\log (z) \sim(z-1)$ as $z \rightarrow 1$, (2.12), (2.18), (2.24),

(2.28) and the continuous mapping theorem yield

$$
\begin{aligned}
& \frac{1}{\tilde{\tau}_{n}^{(1)}} \sum_{j=0}^{k-1} \log \left(A_{j, k, n}\right) \\
= & \frac{1}{\tilde{\tau}_{n}^{(1)}} \sum_{j=0}^{k-1}\left[\frac{|X|_{n, n-k}}{|X|_{n, n-j}}\left(1-\frac{\gamma|X|_{n, n-k}}{\sigma(n / k)}\right)+\frac{\gamma|X|_{n, n-k}}{\sigma(n / k)}-1\right]\left(1+o_{P}(1)\right) \\
= & \frac{k}{\tilde{\tau}_{n}^{(1)}}\left(\frac{\gamma|X|_{n, n-k}}{\sigma(n / k)}-1\right) \frac{1}{k} \sum_{j=0}^{k-1}\left(1-\frac{|X|_{n, n-k}}{|X|_{n, n-j}}\right)\left(1+o_{P}(1)\right) \\
= & \frac{\gamma}{(\gamma+1)} \frac{\sqrt{k}}{\tilde{\tau}_{n}^{(1)}}\left[\sqrt{k} \frac{\gamma \exp \left(u_{n}\right)}{\sigma(n / k)}\left(\frac{|X|_{n, n-k}}{\exp \left(u_{n}\right)}-1\right)+\sqrt{k}\left(\frac{\gamma \exp \left(u_{n}\right)}{\sigma(n / k)}-1\right)\right]\left(1+o_{P}(1)\right) \\
= & \frac{\gamma}{(\gamma+1)} \frac{k}{\tilde{\tau}_{n}^{(1)}}\left(\frac{|X|_{n, n-k}}{\exp \left(u_{n}\right)}-1\right)\left(1+o_{P}(1)\right) \\
= & \frac{\gamma}{(\gamma+1)} \frac{k}{\tilde{\tau}_{n}^{(1)}}\left(\log \left(|X|_{n, n-k}\right)-u_{n}\right)\left(1+o_{P}(1)\right)=\frac{\gamma^{2} S_{n}^{(1)}\left(u_{n}\right)}{(\gamma+1)}+o_{P}(1)
\end{aligned}
$$

This shows (2.29). The proof of (2.30) is similar by noticing that $1-z^{r / \gamma} \sim$ $r(1-z) / \gamma$ as $z \rightarrow 1$.

Now we are ready to show the joint asymptotic normality of the two tail array sums involved in the likelihood moment estimation (1.3) and (1.4). As usual, the idea is to use the Cramér-Wold device. 
Theorem 5 Let the Assumptions 1-9 hold. Further assume that there exists a positive sequence $w_{n}, w_{n} \rightarrow \infty$ as $n \rightarrow \infty$, such that for some $0<\varepsilon<1 / \gamma \wedge 1$ :

$$
r_{n} w_{n} \exp \left((\varepsilon-1 / \gamma) w_{n}\right) \rightarrow 0
$$

and

$$
\frac{r_{n} w_{n}}{\sqrt{k}} \rightarrow 0
$$

as $n, k, n / k \rightarrow \infty$. Also, denote

$$
\begin{aligned}
& \varsigma_{1}:=\sqrt{k}\left(\frac{1}{k} \sum_{j=0}^{k-1} \log \left(1+\frac{\gamma Y_{j}^{\prime \prime}}{\sigma(n / k)}\right)-\gamma\right), \\
& \varsigma_{2}:=\sqrt{k}\left(\frac{1}{k} \sum_{j=0}^{k-1}\left(1+\frac{\gamma Y_{j}^{\prime \prime}}{\sigma(n / k)}\right)^{r / \gamma}-\frac{1}{(1-r)}\right)
\end{aligned}
$$

and $\varsigma:=\left(\varsigma_{1}, \varsigma_{2}\right)^{T}$. Then

$$
\varsigma \stackrel{D}{\rightarrow} N(\mathbf{0}, \Sigma),
$$

as $n, k, n / k \rightarrow \infty$, where

$$
\Sigma:=\left(\begin{array}{cc}
\kappa_{1}(\gamma, r) & -\kappa_{3}(\gamma, r) \\
-\kappa_{3}(\gamma, r) & \kappa_{2}(\gamma, r)
\end{array}\right),
$$

with $\kappa_{1}(\gamma, r), \kappa_{2}(\gamma, r), \kappa_{3}(\gamma, r)$ given in (2.12)-2.14).

Proof Let $\alpha_{1}, \alpha_{2} \in \mathbb{R} \backslash\{0\}$ and define $\boldsymbol{\alpha}:=\left(\alpha_{1}, \alpha_{2}\right)^{T}$. If we are able to show that

$$
\boldsymbol{\alpha}^{T} \boldsymbol{~} \stackrel{D}{\rightarrow} N\left(0, \boldsymbol{\alpha}^{T} \Sigma \boldsymbol{\alpha}\right),
$$

$n, k, n / k \rightarrow \infty$, the desired result simply follows by the Cramér-Wold device. Recall that $Y_{j}=\log \left(\left|X_{j}\right|\right)-u_{n}, \beta_{1}^{\prime}=\gamma /(\gamma+1), \beta_{2}^{\prime}=-r /(1-r+\gamma)$ and $\tilde{\phi}_{i}(x)=\phi_{i}(x)-\beta_{i}^{\prime} \mathbf{1}\{x>0\}, i=1,2$. Then, by virtue of Result 11 Result 3 , Result 4. (2.27) and the definition of $S_{n}^{(i)}(x), i=1,2$, it is easy to see that

$$
\begin{aligned}
& \varsigma_{1}=\frac{1}{\sqrt{k}}\left(\sum_{j=1}^{n}\left[\tilde{\phi}_{1}\left(Y_{j}\right)-\mathrm{E}\left(\tilde{\phi}_{1}\left(Y_{1}\right)\right)\right]\right)+o_{P}(1), \\
& \varsigma_{2}=-\frac{1}{\sqrt{k}}\left(\sum_{j=1}^{n}\left[\tilde{\phi}_{2}\left(Y_{j}\right)-\mathrm{E}\left(\tilde{\phi}_{2}\left(Y_{1}\right)\right)\right]\right)+o_{P}(1),
\end{aligned}
$$

Thus, writing $\tilde{\phi}(x):=\alpha_{1} \tilde{\phi}_{1}(x)-\alpha_{2} \tilde{\phi}_{2}(x)$, the left hand side of (2.35) may be rewritten as

$$
\boldsymbol{\alpha}^{T} \boldsymbol{\varsigma}=\frac{\tilde{\tau}_{n}}{\sqrt{k}}\left(\frac{1}{\tilde{\tau}_{n}} \sum_{j=1}^{n}\left[\tilde{\phi}\left(Y_{j}\right)-\mathrm{E}\left(\tilde{\phi}\left(Y_{1}\right)\right)\right]\right)+o_{P}(1)
$$


where $\tilde{\tau}_{n}^{2}$ is the block-sum variance of the tail array sum with function $\tilde{\phi}$, i.e.

$$
\tilde{\tau}_{n}^{2}:=\frac{n}{r_{n}} \operatorname{Var}\left(\sum_{j=1}^{r_{n}} \tilde{\phi}\left(Y_{j}\right)\right)=\boldsymbol{\alpha}^{T} \Sigma_{n} \boldsymbol{\alpha}
$$

with

$$
\Sigma_{n}:=\left(\begin{array}{cc}
\left(\tilde{\tau}_{n}^{(1)}\right)^{2} & -\tilde{\tau}_{n}^{(1,2)} \\
-\tilde{\tau}_{n}^{(1,2)} & \left(\tilde{\tau}_{n}^{(2)}\right)^{2}
\end{array}\right) .
$$

From (2.12)-(2.14), we simply conclude that $\tilde{\tau}_{n} / \sqrt{k}=\sqrt{\boldsymbol{\alpha}^{T} \Sigma_{n} \boldsymbol{\alpha} / k} \rightarrow \sqrt{\boldsymbol{\alpha}^{T} \Sigma \boldsymbol{\alpha}}$ as $n, k, n / k \rightarrow \infty$ and hence, by a simple application of Slutsky's Theorem on (2.36), the proof is complete if we are able to show that for $n, k, n / k \rightarrow \infty$ :

$$
\frac{1}{\tilde{\tau}_{n}} \sum_{j=1}^{n}\left[\tilde{\phi}\left(Y_{j}\right)-\mathrm{E}\left(\tilde{\phi}\left(Y_{1}\right)\right)\right] \stackrel{D}{\rightarrow} N(0,1) .
$$

But this just means that we need to check that all the conditions up to the Lindeberg Condition of Theorem 4.1 in Rootzén et al. (15]) are satisfied for the function $\psi(x):=\left(\tilde{\phi}(x)-\mathrm{E}\left(\tilde{\phi}\left(Y_{1}\right)\right)\right) / \tilde{\tau}_{n}$. Clearly, their basic Assumptions and their (4.1) hold due to our Assumptions 5-7 and $\psi$ satisfies their (4.2) by definition. Furthermore, their negligibility conditions (2.3) hold by Lemma 6 below and their Corollary 2.2. so that it only suffices to check whether the Lindeberg Condition holds. Therefore, define $Z:=\tilde{\tau}_{n}^{-1} \sum_{j=1}^{r_{n}}\left[\tilde{\phi}\left(Y_{j}\right)-\mathrm{E}\left(\tilde{\phi}\left(Y_{1}\right)\right)\right], Z_{i}:=$ $\left(\tau_{n}^{(i)}\right)^{-1} \sum_{j=1}^{r_{n}}\left[\phi_{i}\left(Y_{j}\right)-\mathrm{E}\left(\phi_{i}\left(Y_{1}\right)\right)\right], i=1,2$ and $Z_{I}:=\left(\tau_{n}^{(I)}\right)^{-1} \sum_{j=1}^{r_{n}}\left[\mathbf{1}\left\{Y_{j}>0\right\}\right.$ $\left.-\mathrm{P}\left(Y_{j}>0\right)\right]$. Also notice from (2.12) -(2.14) that there always exists a $n_{0}>0$ and a constant $K>0$ such that for any $n>n_{0}: \tau_{n}^{(i)}, \tau_{n}^{(I)} \leq K \tilde{\tau}_{n}, i=1,2$. Using this fact and with the help of their inequality (6.4) which holds for any r.v.s $X, Y$ and $\varepsilon>0$ :

$$
(X+Y)^{2} \mathbf{1}\{|X+Y| \geq \varepsilon\} \leq 4\left(X^{2} \mathbf{1}\{|X| \geq \varepsilon / 2\}+Y^{2} \mathbf{1}\{|Y| \geq \varepsilon / 2\}\right),
$$

we conclude by definition of $\tilde{\phi}$ and denoting $A:=\alpha_{2} \beta_{2}^{\prime}-\alpha_{1} \beta_{1}^{\prime}$ that for any $n>n_{0}$ :

$$
\begin{aligned}
& m_{n} \mathrm{E}\left(Z^{2} \mathbf{1}\{|Z|>\varepsilon\}\right) \\
& =m_{n} \mathrm{E}\left[\left(\alpha_{1} \frac{\tau_{n}^{(1)}}{\tilde{\tau}_{n}} Z_{1}+\left(-\alpha_{2} \frac{\tau_{n}^{(2)}}{\tilde{\tau}_{n}} Z_{2}\right)+A \frac{\tau_{n}^{(I)}}{\tilde{\tau}_{n}} Z_{I}\right)^{2}\right. \\
& \left.\quad \cdot \mathbf{1}\left\{\left|\alpha_{1} \frac{\tau_{n}^{(1)}}{\tilde{\tau}_{n}} Z_{1}+\left(-\alpha_{2} \frac{\tau_{n}^{(2)}}{\tilde{\tau}_{n}} Z_{2}\right)+A \frac{\tau_{n}^{(I)}}{\tilde{\tau}_{n}} Z_{I}\right|>\varepsilon\right\}\right] \\
& \leq 4 m_{n} \mathrm{E}\left(\left(\alpha_{1} \frac{\tau_{n}^{(1)}}{\tilde{\tau}_{n}} Z_{1}+\left(-\alpha_{2} \frac{\tau_{n}^{(2)}}{\tilde{\tau}_{n}} Z_{2}\right)\right)^{2} \mathbf{1}\left\{\left|\alpha_{1} \frac{\tau_{n}^{(1)}}{\tilde{\tau}_{n}} Z_{1}+\left(-\alpha_{2} \frac{\tau_{n}^{(2)}}{\tilde{\tau}_{n}} Z_{2}\right)\right|>\varepsilon / 2\right\}\right)
\end{aligned}
$$




$$
\begin{aligned}
& \quad+4 m_{n}\left(A \frac{\tau_{n}^{(I)}}{\tilde{\tau}_{n}}\right)^{2} \mathrm{E}\left(Z_{I}^{2} \mathbf{1}\left\{\left|Z_{I}\right|>\varepsilon /\left|2 A \frac{\tilde{\tau}_{n}}{\tau_{n}^{(I)}}\right|\right\}\right) \\
& \leq 16 m_{n}\left(\alpha_{1} \frac{\tau_{n}^{(1)}}{\tilde{\tau}_{n}}\right)^{2} \mathrm{E}\left(Z_{1}^{2} \mathbf{1}\left\{\left|Z_{1}\right|>\varepsilon /\left|4 \alpha_{1} \frac{\tilde{\tau}_{n}}{\tau_{n}^{(1)}}\right|\right\}\right) \\
& +16 m_{n}\left(\alpha_{2} \frac{\tau_{n}^{(2)}}{\tilde{\tau}_{n}}\right)^{2} \mathrm{E}\left(Z_{2}^{2} \mathbf{1}\left\{\left|Z_{2}\right|>\varepsilon /\left|4 \alpha_{2} \frac{\tilde{\tau}_{n}}{\tau_{n}^{(2)}}\right|\right\}\right) \\
& \quad+4 m_{n}\left(A \frac{\tau_{n}^{(I)}}{\tilde{\tau}_{n}}\right)^{2} \mathrm{E}\left(Z_{I}^{2} \mathbf{1}\left\{\left|Z_{I}\right|>\varepsilon /\left|2 A \frac{\tilde{\tau}_{n}}{\tau_{n}^{(I)}}\right|\right\}\right) \\
& \leq \text { const. }\left[m_{n} \mathrm{E}\left(Z_{1}^{2} \mathbf{1}\left\{\left|Z_{1}\right|>\varepsilon /\left(4\left|\alpha_{1}\right| K\right)\right\}\right)+m_{n} \mathrm{E}\left(Z_{2}^{2} \mathbf{1}\left\{\left|Z_{2}\right|>\varepsilon /\left(4\left|\alpha_{2}\right| K\right)\right\}\right)\right. \\
& \left.\quad+m_{n} \mathrm{E}\left(Z_{I}^{2} \mathbf{1}\left\{\left|Z_{I}\right|>\varepsilon /(2|A| K)\right\}\right)\right] .
\end{aligned}
$$

But this simply means that we have to check whether the Lindeberg Condition holds for each tail array block-sum with respective function $\left(\phi_{1}(x)-\right.$ $\left.\mathrm{E}\left(\phi_{1}\left(Y_{1}\right)\right)\right) / \tau_{n}^{(1)},\left(\phi_{2}(x)-\mathrm{E}\left(\phi_{2}\left(Y_{1}\right)\right)\right) / \tau_{n}^{(2)}$ and $\left(\mathbf{1}\{x>0\}-\mathrm{P}\left(Y_{1}>0\right)\right) / \tau_{n}^{(I)}$, which is equivalent to check whether all conditions of their Theorem 6.2. are satisfied for the aforementioned functions. Due to our Assumptions 1-3, their (6.1) clearly holds for $\left\{\log \left(\left|X_{j}\right|\right)\right\}$ and so does their (2.4) by virtue of their Lemma 4.3. The other conditions are readily checked so that all terms in (2.37) finally converge to zero.

Lemma 6 Suppose the Assumptions 1-7 hold. Then, using the very same definitions and notations as in the proof of Theorem 5 .

$$
\begin{aligned}
& \frac{n}{r_{n} \tilde{\tau}_{n}^{2}} \operatorname{Var}\left(\sum_{j=1}^{l_{n}} \tilde{\phi}\left(Y_{j}\right)\right) \rightarrow 0, \\
& \tilde{\tau}_{n}^{-2} \operatorname{Var}\left(\sum_{j=1}^{n-r_{n} m_{n}} \tilde{\phi}\left(Y_{j}\right)\right) \rightarrow 0,
\end{aligned}
$$

as $n, k, n / k \rightarrow \infty$.

Proof To prove (2.38), split up the variance into parts of variances and covariances:

$$
\begin{aligned}
& \frac{n}{r_{n} \tilde{\tau}_{n}^{2}} \operatorname{Var}\left(\sum_{j=1}^{l_{n}} \tilde{\phi}\left(Y_{j}\right)\right) \\
= & \frac{n}{r_{n} \tilde{\tau}_{n}^{2}}\left[\alpha_{1}^{2} \operatorname{Var}\left(\sum_{j=1}^{l_{n}} \phi_{1}\left(Y_{j}\right)\right)+\alpha_{2}^{2} \operatorname{Var}\left(\sum_{j=1}^{l_{n}} \phi_{2}\left(Y_{j}\right)\right)+A^{2} \operatorname{Var}\left(\sum_{j=1}^{l_{n}} \mathbf{1}\left\{Y_{j}>0\right\}\right)\right.
\end{aligned}
$$




$$
\begin{aligned}
& -2 \alpha_{1} \alpha_{2} \operatorname{Cov}\left(\sum_{j=1}^{l_{n}} \phi_{1}\left(Y_{j}\right), \sum_{j=1}^{l_{n}} \phi_{2}\left(Y_{j}\right)\right)+2 A \alpha_{1} \operatorname{Cov}\left(\sum_{j=1}^{l_{n}} \phi_{1}\left(Y_{j}\right), \sum_{j=1}^{l_{n}} \mathbf{1}\left\{Y_{j}>0\right\}\right) \\
& \left.-2 A \alpha_{2} \operatorname{Cov}\left(\sum_{j=1}^{l_{n}} \phi_{2}\left(Y_{j}\right), \sum_{j=1}^{l_{n}} \mathbf{1}\left\{Y_{j}>0\right\}\right)\right] .
\end{aligned}
$$

Now, recall that all conditions of Lemma 4.3 in 15 are satisfied for each of the three functions $\left(\phi_{1}(x)-\mathrm{E}\left(\phi_{1}\left(Y_{1}\right)\right)\right) / \tau_{n}^{(1)},\left(\phi_{2}(x)-\mathrm{E}\left(\phi_{2}\left(Y_{1}\right)\right)\right) / \tau_{n}^{(2)}$ and $\left(\mathbf{1}\{x>0\}-\mathrm{P}\left(Y_{1}>0\right)\right) / \tau_{n}^{(I)}$ and since by definition $\tilde{\tau}_{n}$ is a linear combination of $\tau_{n}^{(1)}, \tau_{n}^{(2)}, \tau_{n}^{(I)}, \tau_{n}^{(1,2)}, \tau_{n}^{(I, 1)}$ and $\tau_{n}^{(I, 2)}$ and all elements are asymptotically of the same order with different constants by Result 1, all variance terms above clearly converge to zero as $n, k, n / k \rightarrow \infty$. Then, for the first covariance term:

$$
\begin{aligned}
& \frac{n}{r_{n} \tilde{\tau}_{n}^{2}} \operatorname{Cov}\left(\sum_{j=1}^{l_{n}} \phi_{1}\left(Y_{j}\right), \sum_{j=1}^{l_{n}} \phi_{2}\left(Y_{j}\right)\right) \\
= & \frac{n}{r_{n} \tilde{\tau}_{n}^{2}} \sum_{j=1}^{l_{n}} \operatorname{Cov}\left(\phi_{1}\left(Y_{j}\right), \phi_{2}\left(Y_{j}\right)\right)+\frac{n}{r_{n} \tilde{\tau}_{n}^{2}} \sum_{i<j}^{l_{n}} \operatorname{Cov}\left(\phi_{1}\left(Y_{i}\right), \phi_{2}\left(Y_{j}\right)\right) \\
& +\frac{n}{r_{n} \tilde{\tau}_{n}^{2}} \sum_{i>j}^{l_{n}} \operatorname{Cov}\left(\phi_{1}\left(Y_{i}\right), \phi_{2}\left(Y_{j}\right)\right):=\mathrm{I}_{1}+\mathrm{I}_{2}+\mathrm{I}_{3} .
\end{aligned}
$$

Going along the steps of [13], p. 708-711, it is easy to see that

$$
\begin{aligned}
\mathrm{I}_{1} & =\frac{l_{n}}{r_{n}} \frac{k}{\tilde{\tau}_{n}^{2}} \frac{n}{k} \operatorname{Cov}\left(\phi_{1}\left(Y_{1}\right), \phi_{2}\left(Y_{1}\right)\right) \\
& =o(1) O(1) O(1)=o(1)
\end{aligned}
$$

as $n, k, n / k \rightarrow \infty$ because $l_{n} / r_{n}=o(1), k / \tilde{\tau}_{n}^{2}=O(1)$ and $\frac{n}{k} \operatorname{Cov}\left(\phi_{1}\left(Y_{1}\right), \phi_{2}\left(Y_{1}\right)\right) \sim$ $\frac{n}{k} \mathrm{E}\left(\phi_{1}\left(Y_{1}\right) \cdot \phi_{2}\left(Y_{1}\right)\right)=\int_{0}^{\infty} \frac{n}{k} \mathrm{P}\left(\phi_{1}\left(Y_{1}\right) \phi_{2}\left(Y_{2}\right)>x\right) d x=O(1)$. Also, by the stationarity of $\left\{Y_{j}\right\}_{j \geq 1}$ :

$$
\begin{aligned}
\mathrm{I}_{2} & =\frac{n}{r_{n} \tilde{\tau}_{n}^{2}} \sum_{j=1}^{l_{n}-1}\left(l_{n}-j\right) \operatorname{Cov}\left(\phi_{1}\left(Y_{1}\right), \phi_{2}\left(Y_{j+1}\right)\right) \\
& =\frac{n l_{n}}{r_{n} \tilde{\tau}_{n}^{2}} \sum_{j=1}^{l_{n}-1} \mathrm{E}\left(\phi_{1}\left(Y_{1}\right), \phi_{2}\left(Y_{j+1}\right)\right)+o(1) \\
& =\frac{k}{\tau_{n}^{2}} \frac{l_{n}}{r_{n}} \frac{n}{k} \sum_{j=1}^{l_{n}-1} \mathrm{E}\left(\phi_{1}\left(Y_{1}\right), \phi_{2}\left(Y_{j+1}\right)\right)+o(1) \\
& =O(1) o(1) O(1)+o(1)=o(1),
\end{aligned}
$$


$n, k, n / k \rightarrow \infty$. Similarly, one shows $\mathrm{I}_{3} \rightarrow 0$ as $n, k, n / k \rightarrow \infty$ so that the whole term asymptotically vanishes. Analogous considerations for the other covariance terms conclude the proof of (2.38). To show (2.39), notice that $0<n-r_{n} m_{n}<r_{n}$, which simply yields $n-r_{n} m_{n}=o(n)$. Thus, the proof is straightforward by simply repeating the previous steps.

Now we can state our main theorem:

Theorem 7 Let the Assumptions 1-9 hold and $r<0$. Further suppose there exists a sequence $w_{n}$ such that (2.32) and (2.33) hold. Then

$$
\sqrt{k}\left(\begin{array}{c}
\hat{\gamma}_{L M E}-\gamma \\
\frac{\hat{\sigma}_{L M E}}{\sigma(n / k)}-1
\end{array}\right) \stackrel{D}{\rightarrow} N\left(\mathbf{0}, L \Sigma L^{T}\right)
$$

as $n, k, n / k \rightarrow \infty$, where

$$
L:=\left(\begin{array}{cc}
-\frac{(1-r)(1+\gamma)}{\gamma r} & \frac{(1-r)^{2}(1+\gamma-r)}{r^{2}} \\
\frac{(1+\gamma)}{\gamma r} & -\frac{(1-r)^{2}(1+\gamma-r)}{r^{2}}
\end{array}\right),
$$

and $\Sigma$ as in 2.34).

Proof Recall that $Y_{i}^{\prime \prime}=|X|_{n, n-i}-|X|_{n, n-k}$ and denote $\mathbf{Y}^{\prime \prime}:=\left\{Y_{0}^{\prime \prime}, Y_{1}^{\prime \prime}, \ldots, Y_{k-1}^{\prime \prime}\right\}$. Next define for $x, y>0$ :

$$
\mathbf{Z}\left(\mathbf{Y}^{\prime \prime}, x, y\right):=\left(\begin{array}{c}
\frac{1}{k} \sum_{i=0}^{k-1} \log \left(1+\frac{x Y_{i}^{\prime \prime}}{y \sigma(n / k)}\right)-x \\
\frac{1}{k} \sum_{i=0}^{k-1}\left(1+\frac{x Y_{i}^{\prime \prime}}{y \sigma(n / k)}\right)^{r / x}-1 /(1-r)
\end{array}\right)
$$

Notice that $\mathbf{Z}\left(\mathbf{Y}^{\prime \prime}, \hat{\gamma}_{L M E}, \hat{\sigma}_{L M E} / \sigma(n / k)\right)=(0,0)^{T}$ due to (1.3) and (1.4) and thus, by the mean value theorem of differentiation, we obtain the following system of equations:

$$
\begin{aligned}
& \mathbf{Z}\left(\mathbf{Y}^{\prime \prime}, \gamma, 1\right)-\mathbf{Z}\left(\mathbf{Y}^{\prime \prime}, \hat{\gamma}_{L M E}, \hat{\sigma}_{L M E} / \sigma(n / k)\right) \\
= & \mathbf{Z}\left(\mathbf{Y}^{\prime \prime}, \gamma, 1\right)=-\mathrm{DZ}\left(\mathbf{Y}^{\prime \prime}, \gamma_{n}, \sigma_{n}\right) \cdot(\Delta \gamma, \Delta \sigma)^{T},
\end{aligned}
$$

where $\left(\gamma_{n}, \sigma_{n}\right)^{T}=\alpha_{n}(\gamma, 1)^{T}+\left(1-\alpha_{n}\right)\left(\hat{\gamma}_{L M E}, \hat{\sigma}_{L M E} / \sigma(n / k)\right)^{T}$ for all $n$ with some $\alpha_{n} \in(0,1), \Delta \gamma:=\hat{\gamma}_{L M E}-\gamma, \Delta \sigma:=\hat{\sigma}_{L M E} / \sigma(n / k)-1$ and

$$
\begin{aligned}
& \operatorname{DZ}\left(\mathbf{Y}^{\prime \prime}, x, y\right):= \\
& \left(\begin{array}{c}
\frac{1}{k} \sum_{i=0}^{k-1} \frac{Y_{i}^{\prime \prime}}{y \sigma(n / k)}\left(1+\frac{x Y_{i}^{\prime \prime}}{y \sigma(n / k)}\right)^{-1}-1 \quad-\frac{1}{k} \sum_{i=0}^{k-1} \frac{x Y_{i}^{\prime \prime}}{y^{2} \sigma(n / k)}\left(1+\frac{x Y_{i}^{\prime \prime}}{y \sigma(n / k)}\right)^{-1} \\
\frac{1}{k} \sum_{i=0}^{k-1}\left[\frac{r Y_{i}^{\prime \prime}}{x y \sigma(n / k)}\left(1+\frac{x Y_{i}^{\prime \prime}}{y \sigma(n / k)}\right)^{r / x-1}\right. \\
\left.-\frac{r}{x^{2}}\left(1+\frac{x Y_{i}^{\prime \prime}}{y \sigma(n / k)}\right)^{r / x} \log \left(1+\frac{x Y_{i}^{\prime \prime}}{y \sigma(n / k)}\right)\right]
\end{array}\right) .
\end{aligned}
$$


Using Theorem 1 in 9 and repeating the very same steps as in the proof of their Lemma 3, several applications of the continuous mapping theorem yield

$$
\mathrm{DZ}\left(\mathbf{Y}^{\prime \prime}, \gamma_{n}, \sigma_{n}\right) \stackrel{P}{\rightarrow}\left(\begin{array}{cc}
-\frac{\gamma}{(1+\gamma)} & -\frac{\gamma}{(1+\gamma)} \\
\frac{-r}{(1-r)^{2}(1+\gamma-r)} & \frac{-r}{(1-r)(1+\gamma-r)}
\end{array}\right)
$$

as $n, k, n / k \rightarrow \infty$.

Notice that the matrix in (2.41) is non-singular (remember that $r<0$ ) and hence, by [8], Lemma 5.3.3, it follows that

$$
-\mathrm{DZ}\left(\mathbf{Y}^{\prime \prime}, \gamma_{n}, \sigma_{n}\right)^{-1} \stackrel{P}{\rightarrow}\left(\begin{array}{cc}
-\frac{(1-r)(1+\gamma)}{\gamma r} & \frac{(1-r)^{2}(1+\gamma-r)}{r^{2}} \\
\frac{(1+\gamma)}{\gamma r} & -\frac{(1-r)^{2}(1+\gamma-r)}{r^{2}}
\end{array}\right)
$$

as $n, k, n / k \rightarrow \infty$.

Now, a simple reformulation of (2.40) yields

$$
\sqrt{k}(\Delta \gamma, \Delta \sigma)^{T}=\sqrt{k} \mathbf{Z}\left(\mathbf{Y}^{\prime \prime}, \gamma, 1\right)\left(-\mathrm{D} \mathbf{Z}\left(\mathbf{Y}^{\prime \prime}, \gamma_{n}, \sigma_{n}\right)^{-1}\right)
$$

and thus by (2.42) and Theorem 5.

$$
\sqrt{k} \mathbf{Z}\left(\mathbf{Y}^{\prime \prime}, \gamma, 1\right) \stackrel{D}{\rightarrow} N(\mathbf{0}, \Sigma)
$$

as $n, k, n / k \rightarrow \infty$. The desired result follows by Slutsky's Theorem.

In the last part of this section, we will simplify and generalize the conditions listed in our main theorem as far as possible. First of all notice that if our Assumptions 1-3 hold, then Assumption 8 can be reformulated as follows:

Assume $\mathrm{F}_{X}$ has a derivative $\mathrm{F}_{X}^{\prime}$ on $\mathbb{R} \backslash\left[-t_{0}, t_{0}\right]$ where $t_{0}>0$. Then (A.8) holds if

$$
\lim _{t \rightarrow \infty} \frac{t \mathrm{~F}_{X}^{\prime}(t)}{1-\mathrm{F}_{X}(t)}=\frac{1}{\gamma} .
$$

Next consider the following assumptions for the cdf of the innovations:

Assume there exists $d<1$ such that

$$
\mathrm{E}\left(Z_{1}^{d}\right)<\infty
$$

and that $\mathrm{G}_{Z}$ has a derivative $\mathrm{G}_{Z}^{\prime}$ which is $L_{1}$-Lipschitz, that is for any $y>0$ and some positive constant $C$ :

$$
\int_{0}^{\infty}\left|\mathrm{G}_{Z}^{\prime}(x)-\mathrm{G}_{Z}^{\prime}(x+y)\right| d x<C y .
$$


Finally assume that

$$
\liminf _{n, k, n / k \rightarrow \infty} n / k^{3 / 2}>0 \quad \text { or } \quad \limsup _{n, k, n / k \rightarrow \infty} n / k^{3 / 2}<\infty . \quad \text { (2.46) ?limsup? }
$$

From 13, pp. 712-713, we derive that if our Assumptions 1 and 2 as well as (2.43)-(2.46) hold, then our Assumptions 5-7 as well as (2.32) and (2.33) are always satisfied. Let's summarize this in the following corollary:

Corollary 8 Let the Assumptions 1 -4 and 9 as well as 2.43-2.46 hold. Then for $r<0$ :

$$
\sqrt{k}\left(\begin{array}{l}
\hat{\gamma}_{L M E}-\gamma \\
\frac{\hat{\sigma}_{L M E}}{\sigma(n / k)}-1
\end{array}\right) \stackrel{D}{\rightarrow} N\left(\mathbf{0}, L \Sigma L^{T}\right)
$$

as $n, k, n / k \rightarrow \infty$.

\section{Checking the conditions of the main theorem}

Even tough Corollary 8 helps for a better understanding under what circumstances bivariate asymptotic normality holds, it still has some assumptions which are rather uneasy to check. Both the von Mises-Condition (2.43) and the second-order (extended) regular variation conditions give constraints on the tail distribution function (tdf) of the linear process $1-\mathrm{F}_{|X|}$. Since this tdf depends on $1-\mathrm{G}_{Z}$ and $\left\{c_{j}\right\}_{j \geq 0}$ (see (1.1)), it is of practical and statistical advantage to reformulate these unpleasant assumptions using only $1-\mathrm{G}_{Z}$ (possibly adding some other mild conditions). In other words, we want to formulate Corollary 8 under assumptions on the tdf of the innovations.

A very powerful tool to remedy this problem is to do asymptotic expansions as proposed in 11. In their Proposition 4.2.2 (which is an application of their main Theorem 2.5.1) they show that if $1-\mathrm{G}_{Z}$ satisfies some smoothness conditions and is second-order regularly varying, then $1-\mathrm{F}_{|X|}$ obeys the von Mises-Condition and is second-order regularly varying itself! This very nice result is indeed not far away from our target (we also need second-order extended regular variation beside the "normal" second-order regular variation) but in fact, as we will see in Example 1, it is possible to expand the results of 1 in such a manner that all conditions of Corollary 8 are satisfied. Nevertheless it would be of great interest to know whether it is possible to expand the above listed results even to second-order extended regular variation to state a complete and practically implementable theorem for bivariate asymptotic normality of the likelihood moment estimators.

In the following example we show that the assumptions of Corollary 8 hold if the cdf of the innovations is Pareto. Let for convenience $\alpha:=1 / \gamma$ :

Example 1 Assume that $\mathrm{G}_{Z}(x)=1-x^{-\alpha}, x \geq 1$ and $\alpha>2$. Let $X_{n}=$ $\sum_{j=0}^{\infty} c_{j} Z_{n-j}$ be a weakly stationary linear process consisting of a sequence 
of non-negative coefficients $\left\{c_{j}\right\}_{j \geq 0}$, at least one $c_{j}>0$, satisfying our Assumptions 3 and 4. This will also ensure that (2.44) and (2.45) trivially hold and that $\left|X_{n}\right|=X_{n}$. Finally recall that $\mathrm{E}\left(Z_{1}\right)=\alpha /(\alpha-1):=\mu>0$ and that $\operatorname{Var}\left(Z_{1}\right)=\alpha /((\alpha-1)(\alpha-2)):=\sigma^{2}$.

Next we are going to apply Theorem 2.5.1 of [1] twice in order to obtain asymptotic expansions for $1-\mathrm{F}_{X}$ and $\left(1-\mathrm{F}_{X}\right)^{\prime}$ :

Using their notations, take $k=0, m=2$ and $\omega=3$ in the first case and $k=1$, $m=0$ and $\omega=2$ in the second case and notice that $\mathrm{G}_{Z}$ is smoothly varying of index $-\alpha$ and order $\omega=3$. Further denote $C_{u}:=\sum_{j=0}^{\infty} c_{j}^{u}$ and assume there exists $0<\xi<1$ such that for $\eta:=\xi(\alpha /(\alpha+3) \wedge 1 / 2)$ :

$$
C_{\eta}<\infty
$$

Also, let

$$
\left(C_{2} C_{\alpha}-C_{\alpha+2}\right) \sigma^{2}+\left(C_{1}^{2} C_{\alpha}-2 C_{1} C_{\alpha+1}+C_{\alpha+2}\right) \mu^{2} \neq 0 .
$$

Then for $t \rightarrow \infty$

$$
\begin{aligned}
1-\mathrm{F}_{X}(t)= & C_{\alpha} t^{-\alpha}+\alpha \mu\left(C_{1} C_{\alpha}-C_{\alpha+1}\right) t^{-\alpha-1}+\frac{1}{2} \alpha(\alpha+1) \cdot \\
& {\left[\left(C_{2} C_{\alpha}-C_{\alpha+2}\right) \sigma^{2}+\left(C_{1}^{2} C_{\alpha}-2 C_{1} C_{\alpha+1}+C_{\alpha+2}\right) \mu^{2}\right] t^{-\alpha-2} } \\
& +o\left(t^{-\alpha-2}\right) \\
:= & \tilde{c}_{1} t^{-\alpha}+\tilde{c}_{2} t^{-\alpha-1}+\tilde{c}_{3} t^{-\alpha-2}+o\left(t^{-\alpha-2}\right)
\end{aligned}
$$

where $\tilde{c}_{1}, \tilde{c}_{3} \neq 0$, and

$$
\left(1-\mathrm{F}_{X}(t)\right)^{\prime}=-\alpha C_{\alpha} t^{-\alpha-1}+o\left(t^{-\alpha-1}\right) .
$$

Now, from (3.1) and (3.2), it simply follows that

$$
\lim _{t \rightarrow \infty} \frac{t \mathrm{~F}_{X}^{\prime}(t)}{1-\mathrm{F}_{X}(t)}=\lim _{t \rightarrow \infty} \frac{t \alpha C_{\alpha} t^{-\alpha-1}(1+o(1))}{C_{\alpha} t^{-\alpha}(1+o(1))}=\alpha,
$$

so that the von Mises-Condition (2.43) obviously holds.

In order to check whether the second-order extended regular and secondorder regular variation assumptions hold, we need an asymptotic expansion for $b(x):=\left(1 /\left(1-\mathrm{F}_{X}\right)\right)^{\leftarrow}(x)$. After some calculations, we deduce that

$$
b(x)=a_{1} x^{1 / \alpha}+a_{2}+a_{3} x^{-1 / \alpha}+o\left(x^{-1 / \alpha}\right),
$$

where

$$
a_{1}=\tilde{c}_{1}^{1 / \alpha}, \quad a_{2}=\tilde{c}_{2} /\left(\alpha \tilde{c}_{1}\right), \quad a_{3}=-\tilde{c}_{1}^{-1 / \alpha-2}\left[(1+\alpha) \tilde{c}_{2}^{2} /(2 \alpha)-\tilde{c}_{1} \tilde{c}_{3}\right] / \alpha
$$

and because we want $a_{3}$ to be non-zero we also assume that

$$
(1+\alpha) \tilde{c}_{2}^{2} /(2 \alpha)-\tilde{c}_{1} \tilde{c}_{3} \neq 0 .
$$


Using (3.3), it is now easy to see that $b(t)$ is $2 \operatorname{ERV}\left(-2 / \alpha, 2 a_{1}^{-1} a_{3} t^{-2 / \alpha} / \alpha\right)$ and our $k$ has to be chosen such that for $n, k, n / k \rightarrow \infty$ :

$$
\sqrt{k} A(n / k)=\text { const. } \sqrt{k}(n / k)^{-2 / \alpha} \rightarrow 0 .
$$

To show that $1-\mathrm{F}_{X}$ is also second-order regularly varying, we have to deal with two cases:

If $\tilde{c}_{2}=0$, then, according to section $2,1-\mathrm{F}_{X}$ is $2 \mathrm{RV}\left(-2,-2 \tilde{c}_{3} t^{-2} / \tilde{c}_{1}\right)$. Hence the rate of convergence is the same as for second-order extended regular variation and the only constraints on our sequence $k$ are (2.46) and (3.4).

In case $\tilde{c}_{2} \neq 0$ the rates of convergence required by the two forms of secondorder condition are not the same because in this particular case $1-\mathrm{F}_{X}$ is $2 \mathrm{RV}\left(-1,-\tilde{c}_{2} t^{-1} / \tilde{c}_{1}\right)$ and $k$ has to be chosen such that for $n, k, n / k \rightarrow \infty$ :

$$
\sqrt{k} A^{*}\left(b_{X}(n / k)\right)=\text { const. } \sqrt{k} b_{X}^{-1}(n / k) \sim \text { const. } \sqrt{k}(n / k)^{-1 / \alpha} \rightarrow 0 .
$$

(3.5) ?convspeed3?

Since every choice of $k$ which fulfils (3.5) automatically satisfies (3.4), the only constraints on our sequence $k$ in case that $\tilde{c}_{2} \neq 0$ are those in (2.46) and (3.5). Thus, a suitable choice of $k$ is

$$
k=k(n):= \begin{cases}n^{2 \theta /(2+\alpha)} & \text { if } \tilde{c}_{2} \neq 0 \\ n^{4 \theta /(4+\alpha)} & \text { if } \tilde{c}_{2}=0\end{cases}
$$

where $\theta \in(0,1)$ is an arbitrary constant.

Hence, assuming the mild conditions (i)-(iv) to hold, all assumptions of Corollary 8 are satisfied for this very special setup.

\section{Acknowledgments}

The authors want to thank William P. McCormick for his contributions and very thorough explanations regarding the asymptotic tail expansions. 


\section{References}

Barbe 1. Barbe, P., McCormick, W.P.: Asymptotic Expansions for Infinite Weighted Convolutions of Heavy Tail Distributions and Applications. Mem. Am. Math. Soc. 922(4), Vol. 197 (2009)

Brockwell 2. Brockwell, P., Davis, R.: Time Series: Theory and Methods, 2nd edition. Springer, New York (1991)

Datta 3. Datta, S., McCormick, W.P.: Inference for tail parameters of a linear process with heavy tail innovations. Ann. Inst. Statist. Math. 50, 337-359 (1998)

deHaan 4. Haan, L. de, Ferreira, A.: Extreme Value Theory. Springer, New York (2006)

Hosking 5. Hosking, J.R.M., Wallis, J.R.: Parameter and Quantile Estimation for the Generalized Pareto Distribution. Technometrics 29, 339-349 (1987)

Hsing 6. Hsing, T.: On tail estimation using dependent data. Ann. Statist. 19, 1547-1569.

Huesler 7. Hüsler, J., Li, D., Raschke, M.: Estimation for the Generalized Pareto Distribution using Maximum Likelihood and Goodness-of-fit. Comm. Statist. Theory and Methods. 40, 2500$2510(2011)$

Lehmann 8. Lehmann, E.L.: Elements of Large-Sample Theory. Springer, New York (1999)

Martig 9. Martig, L., Hüsler, J.: On consistency of the likelihood moment estimators for a linear process with regularly varying innovations. Preprint: http://arxiv.org/abs/1507.03429

Neves 10. Neves, C.: From extended regular variation to regular variation with application in extreme value statistics. J. Math. Anal. Appl. 355(1), 216-230 (2009)

Pickands 11. Pickands, J.: Statistical Inference using Extreme Order Statistics. Ann. Statist. 3, 119131 (1975)

Resnick3 12. Resnick, S.: Heavy-Tail Phenomena. Probabilistic and Statistical Modeling. Springer, New York (2007)

Resnick4 13. Resnick, S., Stâricâ, C.: Asymptotic behaviour of Hill's Estimator for Autoregressive Data. Comm. Statist. Stoch. Models 13(4), 703-721 (1997)

Rootzen1 14. Rootzén, H., Leadbetter, M.R., Haan, L. de: Tail and quantile estimation for strongly mixing stationary sequences. Technical Report 292, Center for Stochastic Processes, Department of Statistics, University of North Carolina, Chapel Hill, NC 27599-3260 (1990)

Rootzen2 15. Rootzén, H., Leadbetter, M.R., Haan, L. de: On the distribution of tail array sums for strongly mixing stationary sequences. Ann. Appl. Probab. 8(3), 868-885 (1998)

Smith 16. Smith, R.L.: Threshold Methods for Sample Extremes. In: Statistical Extremes and Applications, J. Tiago de Oliveira (Ed.). D. Reidel, Dordrecht, 621-638 (1984)

Zhang 17. Zhang, J.: Likelihood Moment Estimation for the Generalized Pareto Distribution. Aust. N.Z. J. Stat. 49, 69-77 (2007) 